\title{
Erratum to: Label-Free Quantitative Proteomic Profiling Identifies Potential Active Components to Exert Pharmacological Effects in the Fruit of Alpinia oxyphylla by Mass Spectrometry
}

\author{
Yakun Dai ${ }^{1} \cdot \operatorname{Lin}_{\text {Yuan }}{ }^{1} \cdot$ Jinxing Fu ${ }^{1} \cdot$ Bingmiao Gao $^{1}$
}

Published online: 6 August 2020

(c) Korean Society of Plant Biologist 2020

\section{Erratum to: Journal of Plant Biology https://doi.org/10.1007/s12374-020-09251-9}

Unfortunately Fig. 2 is incorrect. The correct Fig. 2 is given below.

The original article can be found online at https://doi.org/10.1007/ s12374-020-09251-9.

Bingmiao Gao

gaobingmiao@hainmc.edu.cn

1 Key Laboratory of Tropical Translational Medicine of Ministry of Education, Hainan Key Laboratory for Research and Development of Tropical Herbs, School of Pharmacy, Hainan Medical University, Haikou 571199, People's Republic of China
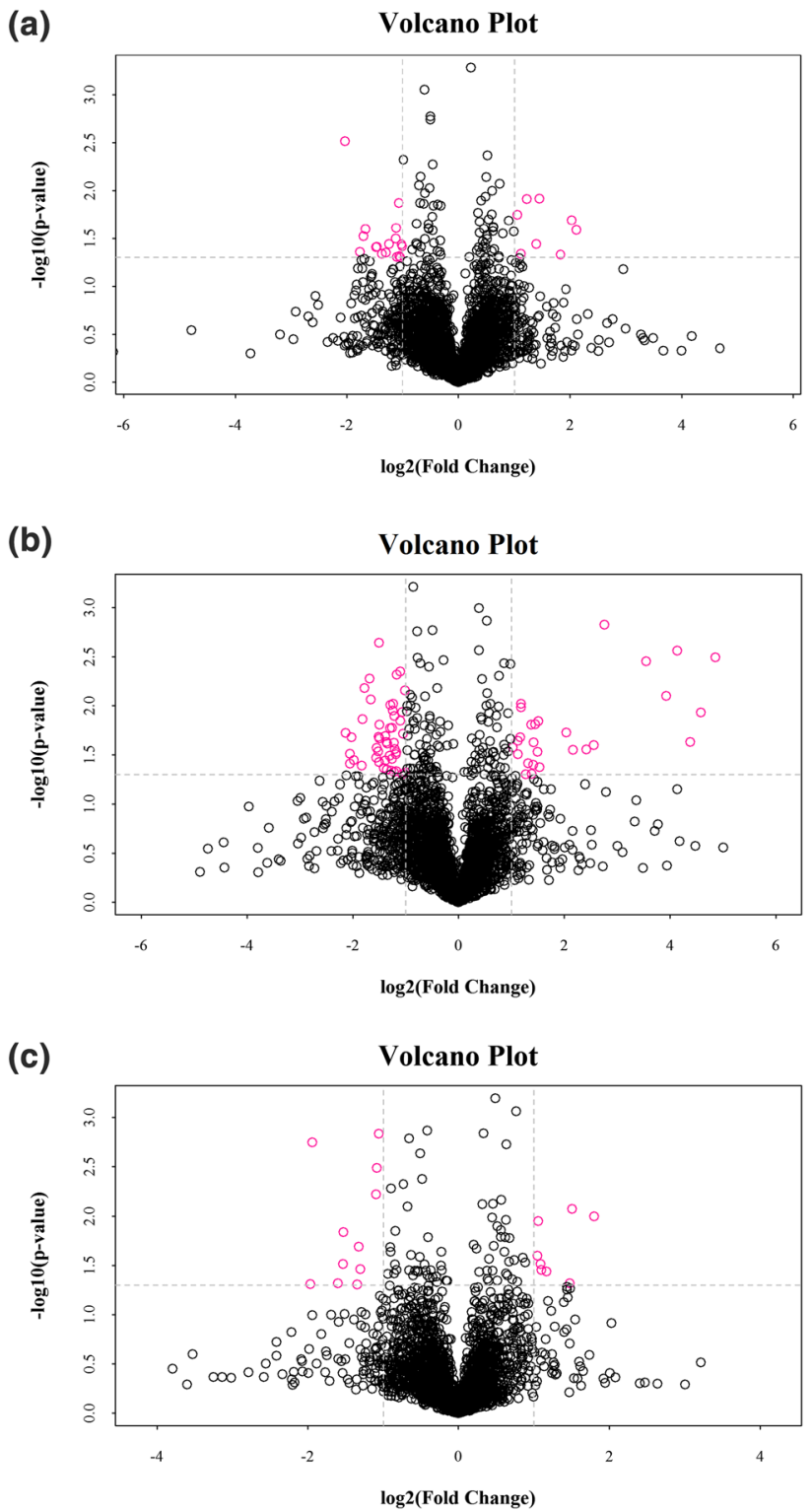

Fig. 2 Volcano plots showing the fold change of differential proteins identified between two A. oxyphylla fruit groups. a The Metaphase group vs the Earlystage group; b the Advanced group vs the Earlystage group; $\mathbf{c}$ the Advanced group vs the Metaphase group 